\title{
Laceração canalicular: uma técnica simplificada de sutura
}

\author{
Canalicular laceration: a simplified suture technique
}

Juliana Vendramini Rossi

Marilisa Nano Costa ${ }^{2}$

\section{R E S U M O}

Os autores descrevem a utilização do cateter de teflon como molde intracanalicular visando tornar a sutura do canalículo lacrimal mais simples e acessível a todo oftalmologista. A técnica consiste em identificar os cotos canaliculares e, após introdução de cateter de teflon (número 22 ou 24) no lúmem canalicular, realizam-se três pontos cardinais transfixantes de sutura do canalículo com o fio nylon monofilamento (Mononylon ${ }^{\circledR}$ ) ou poliglactina trançada $\left(\mathrm{Vicryl}^{\circledR}\right)$ 8-0. Esta técnica está indicada em casos de lacerações regulares, que possibilitem a cateterização do canalículo.

Descritores:Pálpebras/lesões;Aparelholacrimal/cinurgia;Silicones;Técnicasdesutura; Ductonasolacrimal/lesões;Métodos;Intubação

I N T R O D U Ç ̃̃ O

As lacerações canaliculares são as lesões traumáticas mais comuns das vias lacrimais. O diagnóstico correto e tratamento adequado destas lesões são necessários a fim de prevenir epífora pós-traumática ${ }^{(1-2)}$.

Resultados de trabalhos têm indicado variações nas taxas de drenagem lacrimal entre indivíduos e entre os 2 olhos do mesmo indivíduo. Além disso, a taxa de contribuição da drenagem lacrimal pelo canalículo superior e inferior é extremamente variável, fato que não pode ser previsto em cada indivíduo ${ }^{(3)}$.

Não há nenhum teste confiável que permita dizer qual paciente ficará sintomático após a laceração canalicular. Sendo assim, a única maneira de se evitar pacientes sintomáticos seria reparar as lacerações, tanto superiores quanto inferiores ${ }^{(3-4)}$.

Preconiza-se a realização da cirurgia no prazo de 12 a 48 hs para evitar o edema local, facilitando o manuseio cirúrgico ${ }^{(3-4)}$.

O princípio fundamental no reparo de canalículos lacerados consiste em restabelecer a função de drenagem. Diversas técnicas cirúrgicas têm sido descritas nos últimos anos ${ }^{(3,5-6)}$. O desenvolvimento de materiais para reparação e entubação dos canalículos, refinamento de técnicas cirúrgicas e utilização do microscópio, são avanços que contribuem para um melhor prognóstico $^{(3,7-8)}$.

A maioria das técnicas utiliza modeladores de aço inoxidável ou de silicone $^{(3)}$. O silicone é o material mais freqüentemente utilizado para entubação dos canalículos através de várias técnicas que utilizam, por exemplo, a sonda de Worst ou sondas lacrimais que podem ser de vários tipos ${ }^{(1)}$.

Embora a laceração canalicular seja relativamente freqüente, persistem controvérsias quanto à indicação cirúrgica e à técnica ideal ${ }^{(1)}$. 
Adenis (1982) apresentou resultados satisfatórios utilizando sutura com monofilamento sem entubação do canalículo ${ }^{(4)}$.

Objetivando tornar a sutura canalicular uma cirurgia mais simples e acessível a todo oftalmologista, apresenta-se uma nova técnica onde se utiliza o cateter de teflon como molde intracanalicular (Figura 1).

TÉCNICA C IRÚRGICA

Utiliza-se anestesia local (bloqueio do nervo nasociliar) no paciente adulto e anestesia geral na criança.

Emprega-se microscópio cirúrgico para facilitar a identificação dos cotos proximal e distal do canalículo lacerado.

Coloca-se um fio de tração Vicryl ${ }^{\circledR}$ (5-0) no tarso entre as bordas da ferida para permitir a sua aproximação, facilitando a sutura do canalículo, com o fio de nylon monofilamento $\left(\right.$ Mononylon $^{\circledR}$ ) ou poliglactina trançada $\left(V\right.$ icryl $\left.{ }^{\circledR}\right)$. Identificados os cotos do canalículo, introduz-se um catéter de teflon (número 22 ou 24) no lúmem canalicular (Figura 2). Antes de introduzí-lo deve-se cortar o bizel da agulha com a pinça Kelly e mantê-la dentro do catéter durante todo procedimento.

A sutura é realizada em 4 planos:

Canalículo: são passados 3 pontos cardinais transfixantes 8-0 que propiciam maior firmeza da sutura (Figuras 3 e 4).

Conjuntiva: realiza-se sutura contínua com o fio de poliglactina trançada Vicryl ${ }^{\circledR}$ (6-0) na conjuntiva tarsal, sempre que necessário.

Músculo: 2 ou 3 pontos realizados com o fio de poliglactina trançada Vicryl ${ }^{\circledR}(6-0)$ fecham o plano muscular.

Pele: Faz-se sutura com nylon monofilamento preto 6-0 (Mononylon ${ }^{\circledR}$ ) em pontos separados. Esta sutura pode ser feita aproveitando-se o fio de poliglactina trançada 6-0 que foi usado anteriormente, tendo-se o cuidado de retirá-lo após 7 dias, a fim de se evitar hiperpigmentação do local.

Ao final da cirurgia o cateter é removido e nenhum outro modelador é mantido no lúmem canalicular.

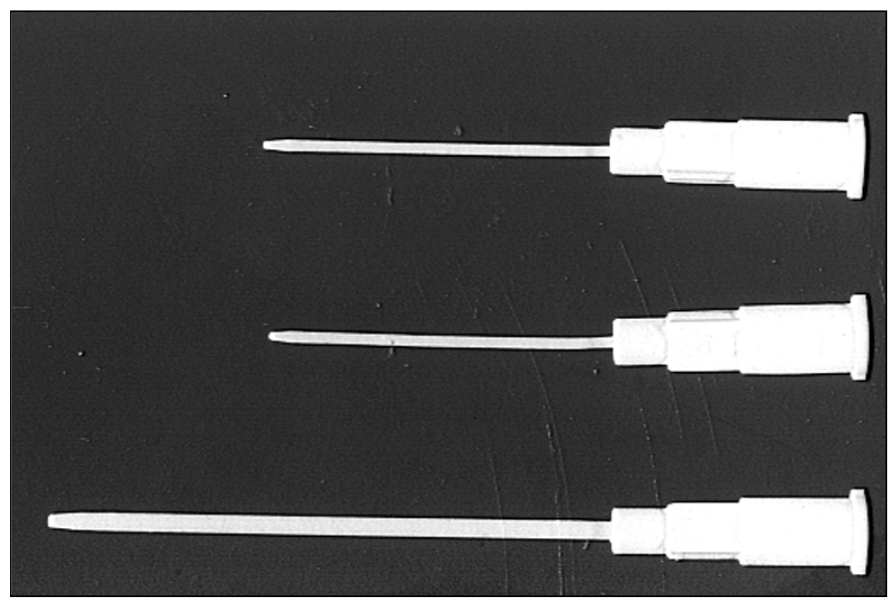

Figura 1-Cateter22 (acima)e cateter 24 (abaixo)

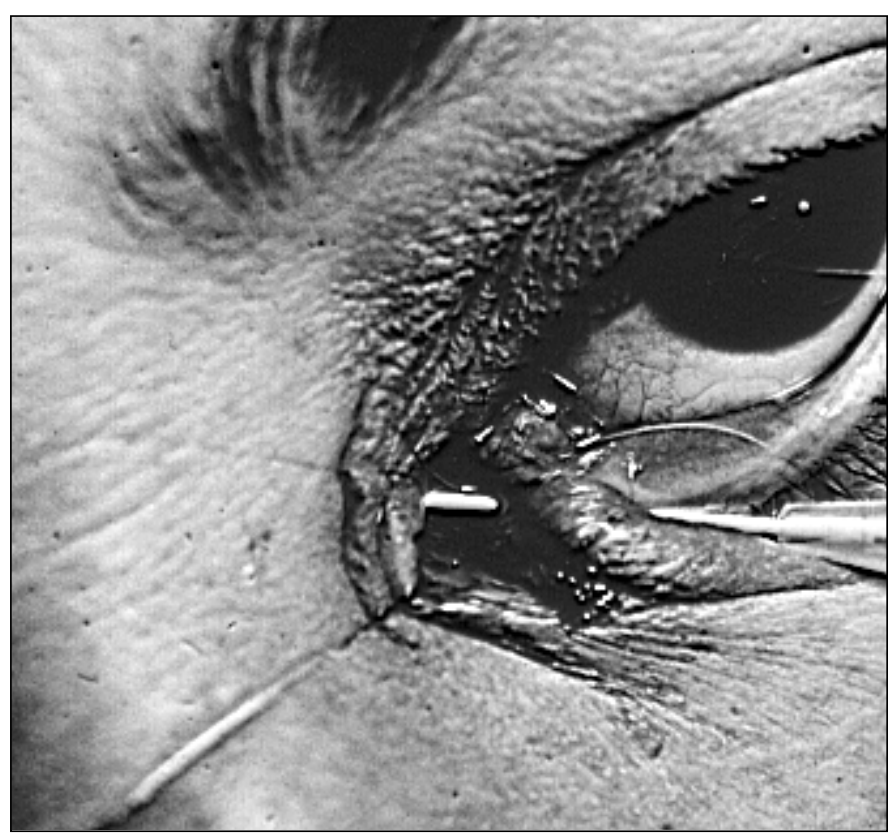

Figura 2-Cateterintroduzidonos cotos proximaledistal docanalículo inferior

CASUÍSTICA

Durante os anos de 1998 e 1999 foram identificados 11 casos de laceração canalicular lacrimal pós-traumática no serviço de oftalmologia da Unicamp que foram operados utilizando-se a técnica descrita neste trabalho. A média de idade foi de 28 anos (variando de 13 anos a 44 anos), sendo 8 do sexo masculino e todos com lesão canalicular inferior. A média de seguimento pós-operatório foi de 6 meses (variando de 3 a 13 meses). Durante este período foi observado patência do canalículo operado em 8 dos 11 casos $(72.7 \%)$, de acordo com a observação clínica e o teste de Jones. Não houve nenhuma complicação intra ou pós-operatória.

\section{I S C U S S ÃO}

Desde o primeiro tratamento cirúrgico de laceração de canalículos, descrito em 1913, houve uma evolução no desenvolvimento dos materiais utilizados na entubação das vias lacrimais durante o ato cirúrgico ${ }^{(3)}$.

As sondas rígidas do tipo Remky já foram abandonadas por serem anti-estéticas e perigosas pelo risco de lesar o ponto lacrimal $^{(3)}$.

A sonda espiral de Worst, ainda utilizada amplamente, limita-se à reparação monocanalicular, principalmente do terço interno, sendo contra-indicada nas lesões do saco lacrimal e canalículo comum. No entanto, nem sempre estão disponíveis nas salas de emergência. Além disso, a entubação do canalículo com estes materiais, pode ser um procedimento complexo para cirurgiões inexperientes. Há risco de danificar o canalículo com formação de falsa via pela dificuldade de seu manuseio ${ }^{(1,3,7-8)}$. 


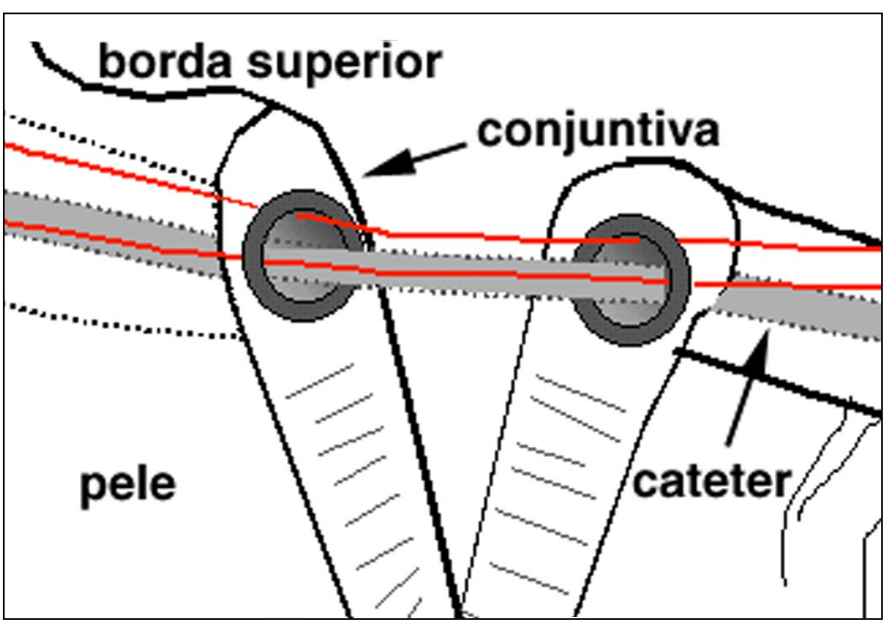

Figura 3-Ilustraçãoda laceraçãocanalicularcomos pontos cardinais desutura

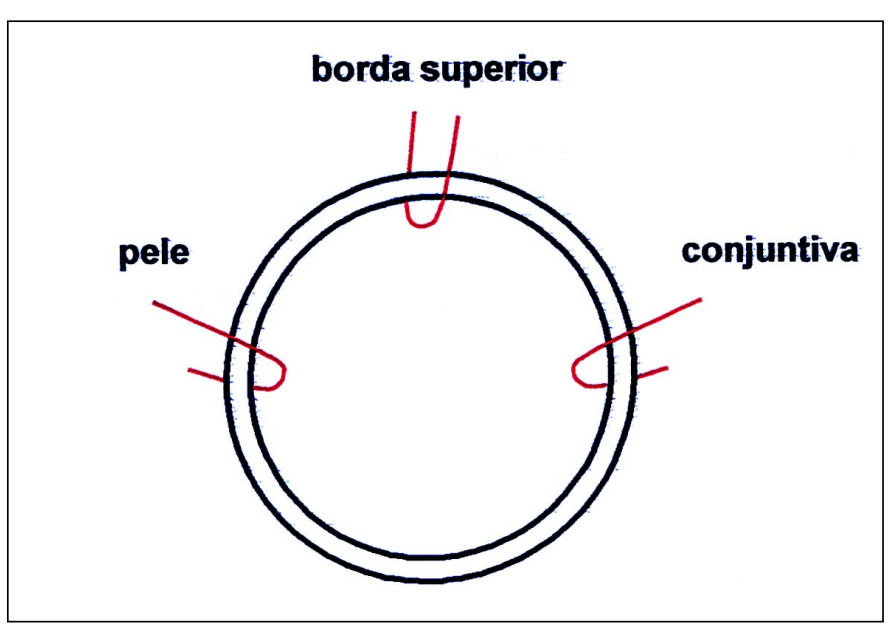

Figura 4-Ilustração do canalículo com os três pontos cardinais de suturatransfixantes

As intubações com silicone bicanaliculo-nasais ou anulares são mantidas de quarenta a sessenta dias e são pouco toleradas pelas crianças, as quais, removem o modelador com freqüência ${ }^{(3)}$. Entre as complicações do uso do silicone na reparação do canalículo encontram-se a formação de granuloma piogênico, inflamação e infecção do canalículo, migração interna ou externa do tubo, irritação nasal crônica e abrasão corneana. Como complicações relacionadas à entubação bicanalículo-nasal vale ressaltar a extrusão do silicone ${ }^{(3,8)}$.

Diante das limitações e possíveis complicações inerentes aos métodos já conhecidos de reparação de lacerações canaliculares, os autores apresentam esta nova técnica utilizando o cateter de teflon como molde intracanalicular somente durante reparação cirúrgica. $\mathrm{O}$ bizel da agulha deve ser removido para que não lese a parede do canalículo e não faça uma falsa via. É importante que a agulha seja mantida dentro do cateter, pois só assim é possível torná-lo mais rígido e de mais fácil introdução no ponto lacrimal. Geralmente são utilizados os cateteres
22 em crianças e 24 em adultos (Figura 1). Acredita-se que esta técnica, além de ser mais prática por empregar material de fácil acesso, é um procedimento pouco traumático e de simples execução. Por ser constituído de um material mais rígido, modela o canalículo, facilitando a sutura sem risco de transfixar o tubo. A nova técnica oferece um menor número de complicações inerentes ao uso de modeladores. Ao final da cirurgia o cateter é removido e nenhum outro modelador é mantido na luz do canalículo. Esta técnica é indicada em todos os casos de lacerações canaliculares regulares, transversais e oblíquas, que possibilitem a cateterização.

Apesar de ser tecnicamente correto a utilização de um tipo específico de fio para cada etapa cirúrgica, acreditamos que com a utilização de apenas 2 tipos de fios de sutura há uma significativa diminuição de custo.

A maior limitação da técnica proposta é a seleção adequada dos pacientes. Devido ao fato de não haver suporte pósoperatório das bordas do canalículo com os "stents", esta técnica fica indicada principalmente nos casos onde o trauma da laceração canalicular não causou perda completa da anatomia das suas bordas. Em casos onde o mecanismo de laceração resulta em bordas muito irregulares esta técnica pode apresentar um índice de patência pós-operatória menor. Entretanto, devido à nossa relativamente baixa casuística e ao relativo curto período de seguimento, não podemos concluir pela sua eficiência ou pela sua incompetência funcional. Maiores estudos devem ser realizados para determinar com maior precisão as suas indicações e os resultados.

A B S T R A C T

The authors describe the use of a teflon catheter as an intracanalicular stent in order to increase its accessability to ophthalmologists. The technique is based on the identification of the canalicular borders followed by the introduction of a teflon catheter ( 22 or 24 ) into the canalicular lumen. Three full-thickness sutures are placed at the canaliculum using a 8-0 mononylon or a 8-0 vicryl. This technique can be used in cases of non-complicated lacerations, in which catheterization is still possible.

Keywords: Eyelids/injuries; Lacrimal apparatus/surgery; Silicones; Suture techniques; Nasolacrimal duct/injuries; Methods; Intubation

REFERE N C I A S

1. Jordan DR, Nerad JA, Tse DT. The pigtail probe, revisited [commented on Ophthalmology 1990;97:1399-400]. Ophthalmology 1990;97:512-9.

2. Loff HJ, Wobig JL, Dailey RA. The bubble test: an atraumatic method for canalicular laceration repair. Ophthal Plast Reconstr Surg 1996;12:61-4.

3. Reifler DM. Management of canalicular laceration [commented on Surv Ophthal 1991;36:323-4]. Surv Ophthalmol 1991;36:113-32.

4. Adenis JP, Robin A. A new method of canalicular surgery: monofilament sutures without intubation. Study of 23 case. J Fr Ophtalmol 1982;5:515-8. 
5. Pingo W, Moribe I, Habib JT, Rodrigues MLV. Lacerações dos canalículos lacrimais. Rev Bras Oftalmol 1992;51:27-30.

6. Bison S, Soccol O. Confecção artesanal de arame de Johnson. Arq Bras Oftalmol 1998;61:599-601.
7. Long JA. A method of monocanalicular silicone intubation. Ophthalmic Surg 1988;19:204-5.

8. Sato K, Kawai K. Repair of canalicular lacerations using silicone tubes $(80$ cases). Nippon Ganka Gakkai Zasshi 2002;106:83-8.

\section{1) (d) XXXII Congresso Brasileiro de Oftalmologia}

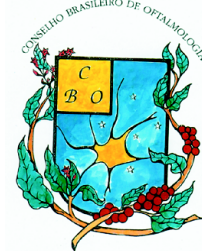

\section{0 a 13 de Setembro de 2003 - Salvador - BA CEN TRO DE CON VEN ÇÕ ES DA BAHIA}

CO N VIDADO S ESTRAN G EIRO S CO N FIRM ADO S
Enique Graue - México
Miguel Burnier - Canadá
Narsing Rao - EUA
Ronald R. Krueger - EUA
Mark J. Mannis - EUA
Todd P. Margolis - EUA
Mark S. Blumenkranz - EUA

CON FERÊN CIA CBO - "RETINA NO SÉCULO XXI" - Dr. Marcos A vila

TAXA DE INSCRIÇÃO

\begin{tabular}{|lccc|}
\hline Categoria & $\begin{array}{c}\text { de } \mathbf{1 6 / 0 4} \\
\text { até } \mathbf{1 6 / 0 6}\end{array}$ & $\begin{array}{c}\text { de } \mathbf{1 7 / 0 6} \\
\text { até } \mathbf{3 1 / 0 7}\end{array}$ & $\begin{array}{c}\text { no } \\
\text { local }\end{array}$ \\
\hline Sócios CBO & $\mathrm{R} \$ 290,00$ & $\mathrm{R} \$ 350,00$ & $\mathrm{R} \$ 460,00$ \\
$\mathrm{~N}$ ão Sócios & $\mathrm{R} \$ 560,00$ & $\mathrm{R} \$ 610,00$ & $\mathrm{R} \$ 720,00$ \\
Residentes/ A cadêmicos & $\mathrm{R} \$ 145,00$ & $\mathrm{R} \$ 175,00$ & $\mathrm{R} \$ 230,00$ \\
Outros Profissionais da Área de Saúde & $\mathrm{R} \$ 145,00$ & $\mathrm{R} \$ 175,00$ & $\mathrm{R} \$ 230,00$ \\
A uxiliaresem O ftalmologia & $\mathrm{R} \$ 75,00$ & $\mathrm{R} \$ 95,00$ & $\mathrm{R} \$ 110,00$ \\
A companhantes & $\mathrm{R} \$ 75,00$ & $\mathrm{R} \$ 95,00$ & $\mathrm{R} \$ 110,00$ \\
\hline
\end{tabular}

A TEN ÇÃ O

DATA - LIM ITE PARA EN VIO DE VÍDEO S: $17 / 06 / 03$

\section{INFO RMAÇÕES:}

SECRETARIA EXECUTIVA / O RGANIZAÇÃO: INTERLN K - Consultoria \& Eventos

Tel.: (71) 336-5644 • Fax: (71) 336-5633

E-mail: oftalmo@interlinkeventos.com.br
AG ÊN CIA DE TURISMO O FICIAL- INTERLINK TURISMO

Tel.: (71) 336-5644 - Fax: (71) 336-5855

E-mail: itl@interlinkeventos.com.br Home Page: www. interlinkeventos.com.br

Home Page: www.interlinkeventos.com.br

CBO : (11) 3266-4000 • Fax: (11) 3171-0953 • E-mail: eventos@ cbo.com.br 